\section{Proximal duodenal obstruction - Bouveret's syndrome revisited}
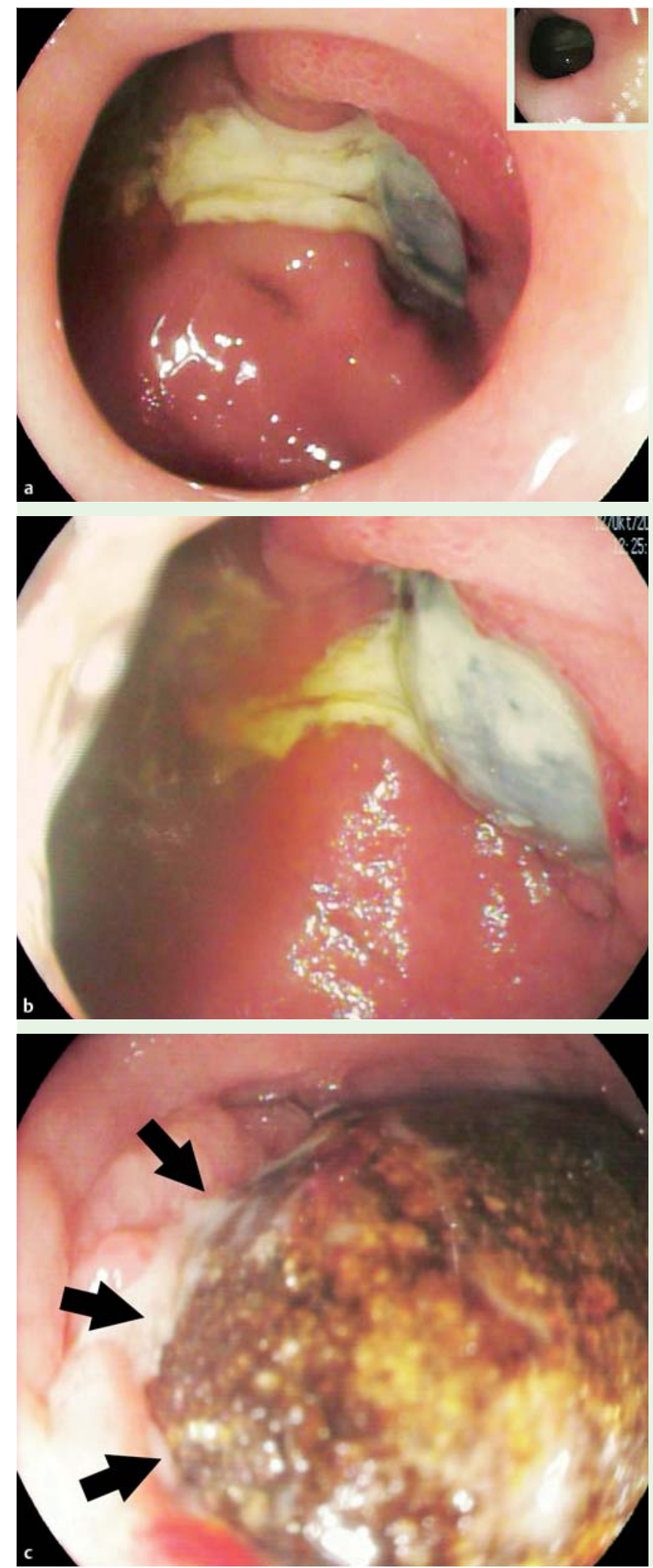

Fig. 1 Endoscopic transpyloric views showing: a the mass that was obstructing the duodenal bulb; b the mass in close-up view; $\mathbf{c}$ the incarcerated gallstone and the orifice of the fistula (arrows), which are visible after thorough flushing of the area.
A 62-year-old man presented to our emergency department with acute epigastric pain and vomiting. He was found to have elevated markers of inflammation with a white cell count of $16.1 \times 10^{9} / \mathrm{L}$ and C-reactive protein (CRP) of $30 \mathrm{mg} / \mathrm{L}$ (normal $<5 \mathrm{mg} / \mathrm{L}$ ), impaired renal function with a creatinine of $1.8 \mathrm{mg} / \mathrm{dL}$, and evidence of mild cholestasis with a bilirubin of $1.3 \mathrm{mg} / \mathrm{dL}$ and $\gamma$-glutamyltransferase (GGT) of 78IU/L.

Esophagogastroduodenoscopy showed grade 3 reflux esophagitis, and $3 \mathrm{~L}$ of gastric fluid were aspirated. Passage of the endoscope beyond the pylorus was obstructed by a mass covered with creamy pus ( Fig. 1 a,b). After the area had been thoroughly flushed, an incarcerated gallstone was found, which was occluding the duodenal bulb with the orifice of the fistula moving relative to the gallstone ( Fig.1c). Endoscopic retrieval (by net, balloon, and snare) failed because of the large diameter of the stone. Computed tomography (CT) scanning revealed an air crescent within the gallbladder and a penetrating gallstone of $3 \mathrm{~cm}$ located within the duodenum, which was completely obliterating the lumen ( $\nabla$ Fig. 2), but there were no signs of intra-abdominal perforation.

During surgery, the penetration of the gallstone through a cholecystoduodenal fistula was confirmed ( $\bullet$ Fig. 3). The large stone could only be removed after fragmentation ( Fig.4). Local excision of the fistula tract was performed and the operation was completed by a cholecystectomy. The patient was discharged from hospital a few days later. On followup 3 months later, he had no specific complaints.

Proximal ileus caused by penetration of a large gallbladder stone is a rare clinical entity that was first described in 1896 by Bouveret [1], but has only rarely been reported since [2]. If technically feasible, endoscopic retrieval and spontaneous regression of the fistula tract have been reported [3] and this approach may be appropriate, especially in older patients or those with comorbidities, if close interdisciplinary follow-up is provided. However, surgical removal of the stone is often the more appropriate solution.

Endoscopy_UCTN_Code_CCL_1AB_2AZ_3AZ

Competing interests: None 


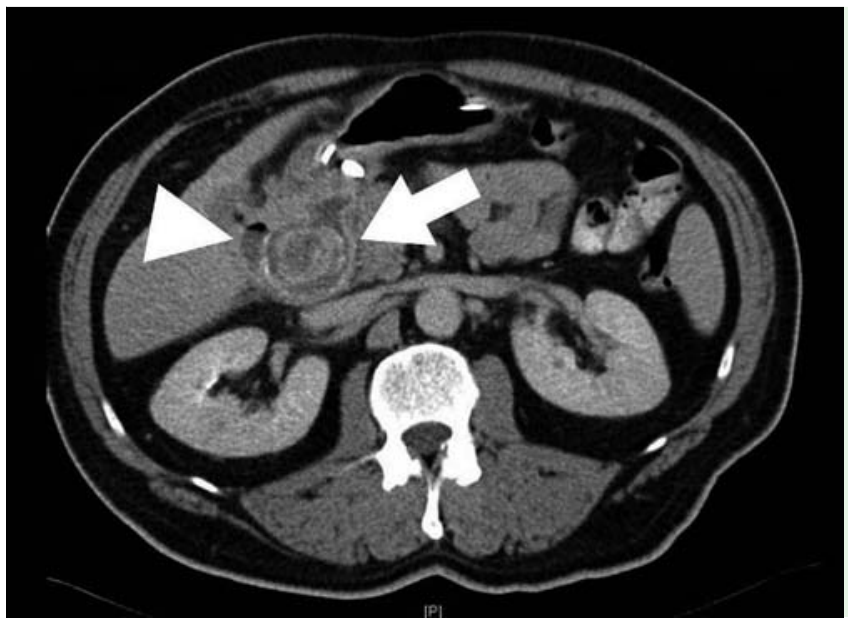

Fig. 2 Computed tomography (CT) scan showing the gallstone that had penetrated into the duodenum (arrow) and an air crescent in the gallbladder (arrowhead).

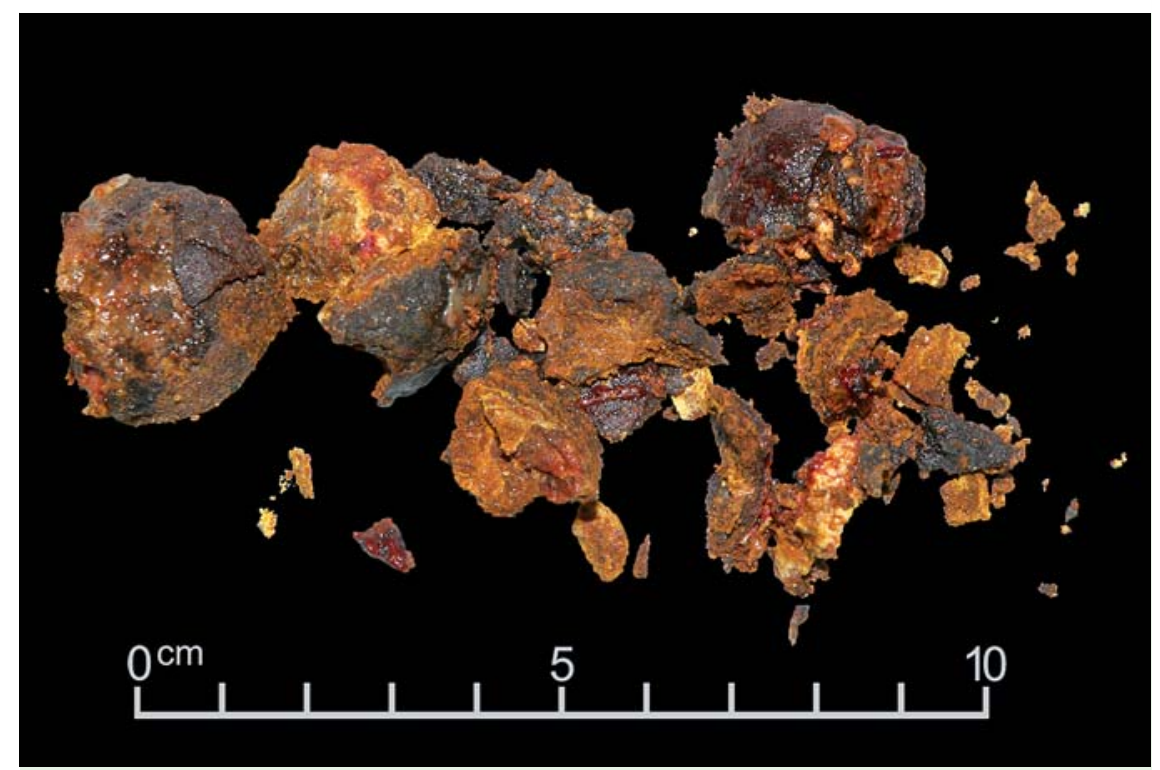

Fig. 4 Photograph of the fragmented gallstone after removal.

C. R. Werner ${ }^{1}$, F. Graepler ${ }^{1}$, J. Glatzle ${ }^{2}$,

D. Stüker², T. Kratt' ${ }^{2}$ J. Schmehl ${ }^{3}$, M. Bitzer ${ }^{1}$, A. Königsrainer²,

\section{N. P. Malek ${ }^{1}$, M. Goetz ${ }^{1}$}

${ }^{1}$ Department of Gastroenterology, Hepatology, and Infectious diseases, University Hospital Tübingen, Medical Clinic, Tübingen, Germany 2 Department of General, Visceral, and Transplant Surgery, University Hospital Tübingen, Tübingen, Germany

${ }^{3}$ Department of Diagnostic and Interventional Radiology, University Hospital Tübingen, Tübingen, Germany

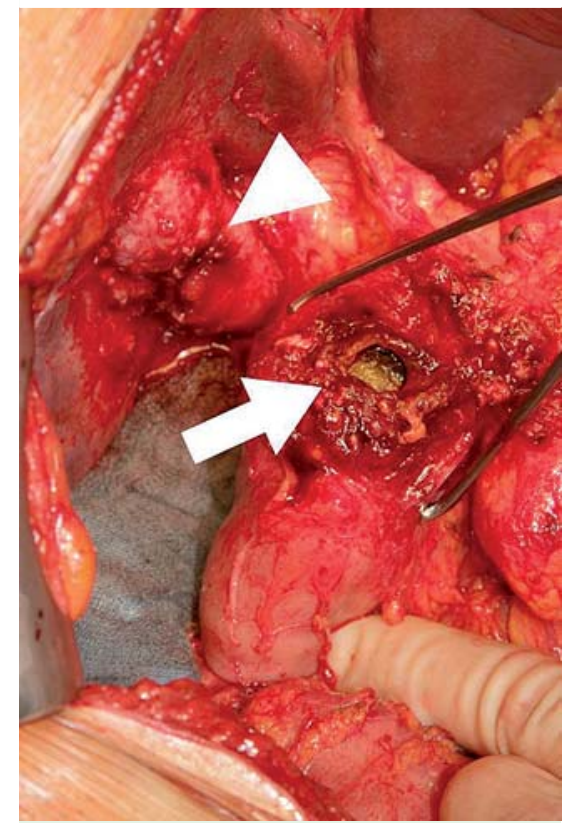

Fig. 3 Photograph taken during surgery showing the cholecystoduodenal fistula with the cystic (arrowhead) and duodenal (arrow) sections visible. The brownish gallstone is also still in situ.
Bibliography

DOI http://dx.doi.org/

10.1055/s-0033-1344324

Endoscopy 2013; 45: E231-E232

(c) Georg Thieme Verlag KG

Stuttgart · New York

ISSN 0013-726X

\section{Corresponding author}

\section{R. Werner, MD}

University Hospital Tübingen, Medical Clinic Department of Gastroenterology, Hepatology, and Infectious diseases

Otfried-Mueller-Str. 10

D-72076 Tübingen

Germany

Fax: +49-7071-295906

christoph.werner@med.uni-tuebingen.de 